\title{
Nanobiosensors as new diagnostic tools for SARS, MERS and COVID-19: from past to perspectives
}

\author{
Riccarda Antiochia ${ }^{1}$ (D)
}

Received: 11 July 2020 / Accepted: 21 October 2020 / Published online: 5 November 2020

(C) The Author(s) 2020

\begin{abstract}
The severe acute respiratory syndrome (SARS), Middle East respiratory syndrome (MERS) and novel coronavirus 19 (COVID19) epidemics represent the biggest global health threats in the last two decades. These infections manifest as bronchitis, pneumonia or severe, sometimes fatal, respiratory illness. The novel coronavirus seems to be associated with milder infections but it has spread globally more rapidly becoming a pandemic. This review summarises the state of the art of nanotechnologybased affinity biosensors for SARS, MERS and COVID-19 detection. The nanobiosensors are antibody- or DNA-based biosensors with electrochemical, optical or FET-based transduction. Various kinds of nanomaterials, such as metal nanoparticles, nanowires and graphene, have been merged to the affinity biosensors to enhance their analytical performances. The advantages of the use of the nanomaterials are highlighted, and the results compared with those obtained using non-nanostructured biosensors. A critical comparison with conventional methods, such as RT-PCR and ELISA, is also reported. It is hoped that this review will provide interesting information for the future development of new reliable nano-based platforms for point-of-care diagnostic devices for COVID-19 prevention and control.
\end{abstract}

Keywords Nanobiosensor - Coronavirus · SARS · MERS · COVID-19 · Point-of-care diagnostics · Immunosensor · DNA-sensor · Nanomaterial

\section{Introduction}

In the last two decades, we have witnessed the outbreak of three zoonotic, highly pathogenic human coronaviruses: severe acute respiratory syndrome coronavirus (SARS-CoV), Middle East respiratory syndrome coronavirus (MERS-CoV) and the 2019 novel coronavirus $(2019-\mathrm{nCoV})$, named as severe acute respiratory syndrome coronavirus 2 (SARS-CoV2) by the International Committee of Taxonomy of Viruses (ICTV) [1-3].

Coronaviruses are a large family of enveloped, singlestranded, positive-sense RNA viruses that mostly infect animals, such as birds and mammals, but may "spill over" from the animal host to human populations. There are seven coronaviruses infecting humans, four of them cause mild infection in the upper respiratory tract, whereas three of them

Riccarda Antiochia

riccarda.antiochia@uniroma1.it

1 Department of Chemistry and Drug Technologies, Sapienza University of Rome, P.le Aldo Moro 5, 00185 Rome, Italy
(SARS-CoV, MERS-CoV, SARS-CoV-2) cause respiratory illnesses of varying severity, from the common cold to fatal pneumonia $[4,5]$. Before SARS appeared, coronaviruses had never been particularly dangerous to humans, causing severe diseases only in animals [6].

According to the World Health Organization (WHO), the onset of the SARS epidemic occurred in Guangdong, China, in November 2002, followed by the worldwide spread of the virus with reported cases in 29 countries, including Canada and the USA. However, 8 months later, in July 2003, after causing 774 deaths, the SARS epidemic was declared to be contained by the WHO [7].

Only a decade later, another pathogenic coronavirus, MERS-CoV, caused an endemic in Middle Eastern countries. Since 2012, there have been at least 845 MERS-CoV-related deaths in 27 countries but about $80 \%$ of the reported cases were in Saudi Arabia. Nowadays, it continues to cause sporadic outbreaks, mostly localized in the Arabian Peninsula [8].

The outbreak of the novel highly contagious SARS-CoV-2 was first identified in Wuhan, Hubei province, China, in early December 2019 [9]. The SARS-CoV-2 virus rapidly spreads across continents and on 30 January 2020, the WHO declared 
the outbreak of a Public Health Emergency of International Concern and a pandemic on 11 March. Infections with SARSCoV-2 are now widespread, and as of 31 August 2020, $25,467.390$ cases have been confirmed in more than 110 countries, with 851,000 deaths [10-13].

The three Coronaviruses belong to the same Betacoronavirus genus [14]. Clinical presentation of COVID-19, the disease caused by SARS-CoV-2 virus, shows great similarities with SARS and MERS pneumonia. More than $80 \%$ of cases are mild, and patients normally recover within 2 weeks. However, some patients show severe symptoms, like acute respiratory distress syndrome (ARDS) and about 5\% show critical conditions, which may evolve into septic shock or multiple organ failure [13].

According to early phylogenetic studies, SARS-CoV-2 is related to SARS and both of them show over $85 \%$ genome sequence identity with the bat SARS-like $\mathrm{CoV}$, which would suggest the bat origin of the virus $[15,16]$.

Several studies hypothesized the entry of these three viruses in humans from their natural reservoir bats, via intermediate host like civets and camels, in the case of SARS-CoV and MERS-CoV, respectively. The intermediate host of the SARS-CoV-2 still needs to be established, although some studies suggest pangolins as a possible host [17].

Although SARS and MERS have significantly higher mortality rates than COVID-19, the novel SARS-CoV-2 is more infectious and the overall number of deaths from COVID-19 far outweighs that from SARS or MERS [18-20]. Table 1 summarises the main features of the three coronaviruses.

Countries are racing to slow the spread of the virus by testing and treating patients, carrying out contact tracing, limiting travel, quarantining citizens, and cancelling large gatherings such as sporting events, concerts and schools [21].

Early diagnostic tests are essential tools to track the spread of the virus in order to control the epidemic. At the moment, most testing for COVID-19 is currently done on viral genetic material from nasopharyngeal swabs, using the reverse transcription polymerase chain reaction (RT-PCR), a molecular biology technique which amplifies a specific genetic sequence of the virus. Alternatively, the enzyme-linked immunosorbent assay (ELISA), a common biochemical technique, is used to detect the specific antibodies or antigens in patient blood. Unfortunately, both methods require expensive instruments and the use of specialized laboratories and well-trained personnel [22]. In particular, serological tests show evidence of low sensitivity, accuracy and specificity [23].
Obviously, the first method tells if a person is currently infected, whereas the second method can determine if a patient has at some point been infected by SARS-CoV-2. Reverse transcription loop-mediated isothermal amplification (RTLAMP) is a more recent molecular technique, where the amplification is conducted at a single temperature and does not need specialized laboratory equipment [24]. However, all these methods are not suitable for point-of-care testing [11, 25].

Therefore, rapid, accurate, low-cost, miniaturized diagnostic platforms for virus detection usable at the point-of-care remain a challenge $[25,26]$. Lateral flow immunoassays are qualitative chromatographic assays, similar to common pregnancy tests, based on a two-site non-competitive format, as ELISA tests, but usable at the point-of-care. They are produced as test kits to be used by a specialist or by the patients themselves, but they suffer from a poor sensitivity [27]. The main characteristics of current methods for COVID-19 detection are summarized in Table 2.

Affinity-based biosensors (ABBs) represent interesting diagnostic tools for early and affordable detection of virus diseases, thanks to their properties, such as high sensitivity, high specificity, fast response time and the possibility of miniaturization for POC use [22, 28-31]. These peculiar characteristics allow them to complement current methods of screening and monitoring of a virus outbreak, especially when in situ and real-time analysis is required.

The recent advances in nanotechnology and the use of nanomaterials in the construction of biosensors resulted in a significant improvement in the performances of these devices [32-34]. Nanomaterials allowed a large increase in biosensors efficiency and sensitivity, thanks to their excellent conductivity, extraordinary photoelectrochemical properties and the possibility of miniaturization of the sensing platform [35-39].

In this review, we describe the nanobiosensors based on affinity interactions reported in the literature for the detection of SARS, MERS and COVID-19. A comparison with nonnanostructured affinity-based biosensors and with conventional methods is also provided. The review is structured into four sections. The first section presents a brief overview of the different classes of nanomaterials used for affinity-based biosensors purposes, and the other sections are divided by analyte type, with a particular focus on COVID-19, because of the urgent need to find early diagnostic methods for SARSCoV-2 detection, in order to deal with the current COVID19 pandemic.

Table 1 Comparative analysis of the main features of SARS-CoV, MERS-CoV and SARS-CoV-2

\begin{tabular}{llllllll}
\hline Disease & Pathogen & Family & Origin & Intermediate & Mortality rate & $\mathrm{R}_{0}$ & Total number of deaths \\
\hline SARS & SARS-CoV & $\beta$ coronaviridiae & Bats & Civets & $9.5 \%$ & 1.8 & 774 \\
MERS & MERS-CoV & $\beta$ coronaviridiae & Bats & Camels & $34.4 \%$ & 0.7 & 858 \\
COVID-19 & SARS-CoV-2 & $\beta$ coronaviridiae & Bats & Pangolins & $2.3 \%$ & $2.0-2.5$ & $>850,000$ \\
\hline
\end{tabular}


Table 2 Main characteristics of current methods for COVID-19 detection

\begin{tabular}{lllll}
\hline Method & Biomarker & Lab or POC & Sample site & Time of analysis \\
\hline RT-PCR & Viral RNA & Lab based & Nasopharyngeal swab & $\sim 4 \mathrm{~h}$ \\
LAMP & Viral RNA & Lab based & Nasopharyngeal swab & $\sim 3 \mathrm{~h}$ \\
Lateral flow & Antibody (or antigen) & POC & Blood & $\sim 15 \mathrm{~min}$ \\
ELISA & Antibody (or antigen) & Lab based & Blood & $\sim 2 \mathrm{~h}$ \\
\hline
\end{tabular}

\section{Affinity-based nanobiosensors}

The use of novel nanomaterials in biosensing may overcome some of the challenges and limitations of biosensor technology. Nanomaterials by definition must have dimensions in the range $1-100 \mathrm{~nm}$. They are developed to exhibit novel characteristics compared to the same material without nanoscale features, such as increased strength, conductivity and unique optical, magnetic, thermal and chemical properties.

Affinity-based nanobiosensors combine the high specificity of the biorecognition agents, namely bioreceptors, such as antibodies, ssDNA and aptamers, with the extraordinary properties of the nanomaterials, which allow enhanced sensitivities and lowered detection limits of several orders of magnitudes [37,40].

The high specific surface interaction of the nanobiosensor with the bioanalyte becomes highly efficient thanks to the extremely large surface/volume ratio, which enables the immobilization of an enhanced amount of bioreceptor units. Thus, the immobilization strategies used to conjugate the biorecognition agents onto the nanomaterials remain a constant challenge [41]. The technique used for the bioreceptor immobilization is one of the key factors in developing a reliable affinity-based nanobiosensor. In addition to the immobilization of the bio-molecules, the nanomaterials can serve for target recognition and for signal transduction and amplification [40].

Various kinds of nanomaterials have been merged to ABBs, such as noble metal nanoparticles, carbon nanostructures, quantum dots and magnetic nanoparticles [34, 35, 42-44].

The ABBs for coronavirus detection reported in the literature are based on gold nanoparticles (AuNPs) and nanoislands (AuNIs), graphene (GR), and nanowires (NWs). Figure 1 shows a schematic diagram of a nanomaterial-based affinity biosensor for coronavirus detection.

\section{Gold nanoparticle and gold nanoisland affinity-based biosensor}

Among the group of metal noble nanoparticles (MNPs), gold nanoparticles (AuNPs) are mostly used in biosensing application for virus infections due to their outstanding optical/ electrical properties, excellent biocompatibility, catalytic properties and relatively simple production pathway [45-47]. MNPs have been widely used as supporting electrode materials increasing electron transfer rates and surface- to-volume ratio, thus allowing the immobilization of large amounts of primary antibodies or cDNA, suppressing the non-specific binding (NSB) of proteins, with a consequent enhancement of the analytical response of the device.

AuNPs play a different role in the biosensing process depending on the transduction mode of the biosensor, namely optical and electrochemical biosensors. There are several optical sensing modalities for AuNPs, surface plasmon resonance (SPR) being the one that attracted most intensive research, as AuNPs are considered to have the ability to amplify the SPR signal [48]. As for the electrochemical biosensors, AuNPs allow to improve the analytical performances of the device through a double mechanism: (i) as novel immobilization platforms/electrochemical transducers, which allow loading of a larger amount of the biosensing element, thanks to the much higher surface area of AuNPs compared to flat gold surfaces; (ii) as labels for signal amplification [40].

AuNPs are often conjugated with other nanomaterials to further improve their binding capacity. In this context, carbon nanotubes (CNTs) have attracted much interest due to their unique properties [49]. Nanohybrids of AuNPs and CNTs have been realized, offering a more effective immobilization matrix. Platforms based on gold nanoislands were also used for numerous sensing applications [50]. They are basically gold aggregates with dimensions in the range $20-80 \mathrm{~nm}$, obtained by deposition and annealing of the AuNPs at high temperature $\left(560^{\circ} \mathrm{C}\right)$ for several hours $(\sim 10 \mathrm{~h})$.

\section{Graphene affinity-based biosensors}

Graphene $(\mathrm{G})$, together with carbon nanotubes (CNTs), is the most promising nanostructured carbon materials for biosensing applications, where each allotrope has its own specific properties and advantages as a transducer element. Carbon nanotubes are one-atom-thick sheets of graphite, called graphene, rolled into cylinders with a diameter of the order of a few nanometres [51-58]. Compared to CNTs, graphene has a much younger history. Graphene and CNTs share some properties in common, including excellent mechanic, electronic and thermal properties [59-61]. Thanks to its defect-rich property, graphene can be easily functionalized by inserting functional groups on the $2 \mathrm{D}$ plane, thus becoming a good support for immobilizing of ligands, such as antibodies and single-strand DNA for developing immunosensors and aptasensors, respectively [43, 62, 63]. 


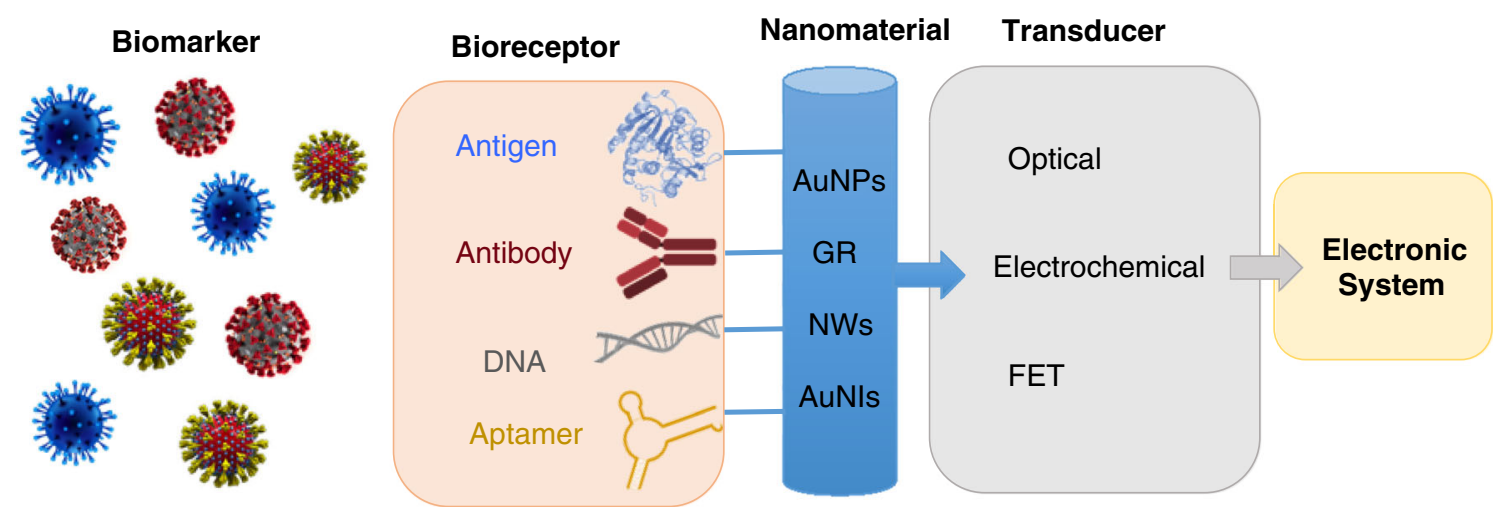

Fig. 1 Schematic diagram of nanomaterial-based affinity biosensor for coronavirus detection. List of abbreviations: AuNPs, gold nanoparticles; GR, graphene; NWs, nanowires; AuNIs, gold nanoislands; FET, field effect transistor

Graphene, as well as CNTs, is water insoluble, which can be overcome by modifying its surface with hydrophilic functional groups in order to increase its solubility and suppress the NSB of proteins onto the electrode surface. Another common strategy to minimize NSB is achieved by graphene functionalization through its oxidation to graphene oxide (GO) and reduced graphene oxide (rGO), which found a lot of applications in immunosensors development [64]. The choice between the use of $\mathrm{G}$ or $\mathrm{GO}$ depends on the need of functionalization of the nanocarbon for biomolecule immobilization (more oxygen groups required) or of a higher conductivity (less oxygen groups required).

As for CNTs, graphene is largely used in electrochemical and optical biosensors or field-effect transistor (FET) setups, where the changes in the conductivity of the graphene channel after the biorecognition event led to high sensitivities [59, 65]. Contrary to CNTs, ssDNA or oligonucleotide bioreceptors are reversibly adsorbed to graphene oxide and successively released after the recognition event, thus allowing the recovering of the bioreceptors [34].

\section{Nanowire affinity-based biosensors}

Nanowires (NWs) are one-dimensional nanostructures in the form of wire that can be composed of both metallic and nonmetallic elements with nanometre sized diameters and micron long lengths. The NWs are robust and have high physical strength, directly attributed to their crystalline structure, and unique 1D morphology, electrical, mechanical, optical, magnetic and thermal properties. Silica NWs are broadly explored for biosensor applications, thanks to their optical, photonic and electronic properties with excellent biocompatibility for sensing application [66, 67].

Silicon and indium oxide NWs are mostly explored as novel biosensing tools for highly sensitive virus detection, due to their wide bandgap, which broadens the scope of detection from purely electrochemical or FET-based detection to more simple optical methods $[68,69]$.

\section{Nanobiosensors for SARS detection}

Up to now, there is only one nanobiosensor for SARS-CoV detection reported in the literature. It is a FET-based immunosensor, developed by Ishikawa and coworkers in 2009 [70], where the antigen-antibody binding generates a change in conductance, correlated to the virus concentration. The biomarker used for SARS-CoV detection is the virus antigen nucleocapsid protein (N-protein), the most abundant protein in coronaviruses. Instead of conventional antibodies, antibody mimic proteins (AMPs) have been utilized as bioreceptors. The AMPs can be easily produced by in vitro selection techniques and are smaller and stable at a wider range of $\mathrm{pH}$ than normal antibodies. A fibronectin-based protein (Fn) has been properly engineered as AMP capture agent. The exposed gate region of the FET-based immunosensor was modified with $\mathrm{In}_{2} \mathrm{O}_{3}$ nanowires on a $\mathrm{Si} / \mathrm{SiO}_{2}$ substrate, in order to improve the immobilization of the AMPs and the signal transducing. The Fn protein was anchored to the NWs via the only thiol functional group present in the whole peptide sequence from a cysteine residue. The schematic diagram showing the covalent immobilization of the Fn probe onto the nanowires is represented in Fig. 2. At the working $\mathrm{pH}=7.4$, the N-proteins are positively charged and therefore their binding on a p-type channel causes depletion of charge carriers (holes) and a consequent decrease in conductance. Bovine serum albumin (BSA) was used as a "blocking agent" for nanowires and source-drain electrodes, thus avoiding NSB, which may lead to false-positive results (Fig. 2). The sodeveloped platform was able to detect the N-protein at subnanomolar concentrations, in the presence of $44 \mu \mathrm{M}$ BSA, with a comparable sensitivity to current immunological detection methods, but with a shorter detection time and without the need of labelled reagents. Indeed, the high sensitivity and high selectivity of the proposed biosensor are achieved by the synergic effect of the $\mathrm{In}_{2} \mathrm{O}_{3}$ nanowires/Fn protein, which is able to selectively detect the SARS biomarker N-protein. Moreover, compared to other metal oxide nanowires $\left(\mathrm{ZnO}, \mathrm{SnO}_{2}\right.$, etc.), 
Fig. 2 Schematic representation of $\operatorname{In}_{2} \mathrm{O}_{3}$ nanowires FET-based immunosensor for SARS-CoV. List of abbreviations: S, source; $\mathrm{D}$, drain; BSA, bovine serum albumin

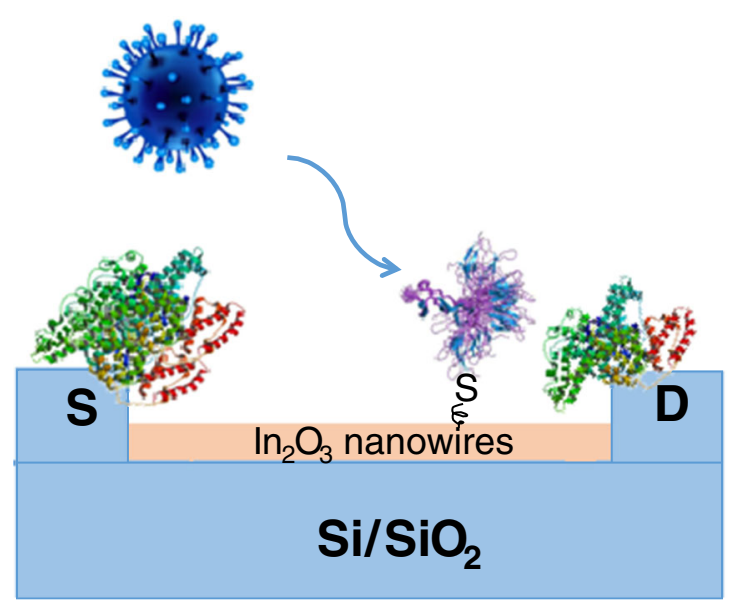

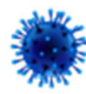

SARS-CoV-2 N-protein

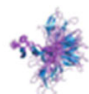

Fibronectin-based protein

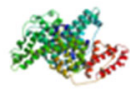

BSA

S thiol
$\mathrm{In}_{2} \mathrm{O}_{3}$ nanowires have the advantage that they do not possess an insulating oxide layer, such as $\mathrm{SiO}_{2}$ for $\mathrm{Si}$ nanowires, which can decrease the sensor sensitivity, and therefore, they contribute to a marked decrease of the detection limit of the sensor.

Table 3 shows the characteristics and the analytical performances of the described SARS immunosensor and a comparison with other "non-nanotechnology-based" immunosensors for SARS detection, reported in the literature. In particular, two SPR-based biosensors [71, 72] and one piezoelectric biosensor [73] have been realized but their sensitivities resulted to be lower of several orders of magnitudes.

\section{Nanobiosensors for MERS detection}

An amperometric nano-immunosensor for MERS-CoV virus detection was described in 2019 by Layqah and Eissa [74]. In this case, the virus spike protein $\mathrm{S} 1$, which is the common target for neutralizing antibodies, was utilized as MERS biomarker [80, 81].

The biosensor is based on an indirect competition between the free virus in the sample and immobilized MERS-CoV recombinant spike protein $\mathrm{S} 1$, for a fixed antibody concentration added to the sample. The immunosensor was realized on an array electrodes system, thus allowing the simultaneous detection of MERS-CoV and $\mathrm{HCoV}$, another human coronavirus. The surface of the carbon electrodes was modified with AuNPs, in order to enhance the electrochemical properties of the electrode, providing a higher surface area and a faster electron transfer rate. Successively, MERS-CoV and $\mathrm{HCoV}$ antigens were immobilized onto the AuNPs/carbon electrode, by a simple drop-casting procedure, after incubating the electrode in a solution containing cysteamine and glutaraldeyde, for covalently binding of the $\mathrm{NH}_{2}$ groups of the antigens. A schematic representation of the AuNPs immunosensor is shown in Fig. 3. The non-specific adsorptions were reduced by incubating the electrode in BSA solution, in order to block the unreacted aldehyde groups and the free gold surface. The experimental conditions were carefully optimized, in particular the concentration of antibody used for incubation of the antigen-modified electrode and the binding time, resulting in $10 \mu \mathrm{g} / \mathrm{mL}$ and $20 \mathrm{~min}$, respectively. The detection was obtained by measuring the peak current signal of the ferro/ ferricyanide redox probe, properly added to the solution, with the square wave voltammetry (SWV) technique. A decrease of the SWV peak current is clearly observed after binding of the antibodies to the immobilized antigens, because of the "coverage" of the electrode surface by the antibody molecules. Thus, a decrease of both electron transfer efficiency and current is registered. The so-realized immunosensor showed a good linear response from 0.001 to $100 \mathrm{ng} / \mathrm{mL}$ for MERS-CoV and a very high sensitivity, with a detection limit of $0.4 \mathrm{pg} / \mathrm{mL}$, definitely lower value than that obtained with ELISA method (1 ng/mL) [82]. The characteristics of the biosensor are summarized in Table 3. The selectivity of the biosensor was studied by using different virus proteins, such as FluA and FluB, showing no cross-reactivity phenomena. The possibility of the use of the proposed biosensor for simultaneous detection of different types of CoVs was also confirmed by mixing the two proteins MERS-CoV and $\mathrm{HCoV}$ on the electrode surface. The stability of the sensor was good, as the sensor showed only $2 \%$ current decrease after 2 weeks. Finally, the proposed immunosensor was successfully tested in spiked nasal samples showing good recovery percentages.

\section{Nanobiosensors for COVID-19 detection}

\section{Viral antigen detection}

The first biosensing strategy is the use of antibodies or cDNA to selectively capture the viral antigen or viral RNA. A graphene-based FET device has been properly engineered by Seo and coworkers to determine SARS-CoV-2 viral load in nasopharyngeal swabs of patients affected by COVID-19, 
thus allowing also the determination of the severity of the COVID-19 disease [75]. The sensing area of the FET-based biosensor is a graphene sheet, transferred to a $\mathrm{SiO}_{2} / \mathrm{Si}$ substrate, and successively modified with SARS-CoV-2 spike antibody, properly immobilized onto the graphene sheet surface by drop-casting, as schematized in Fig. 4. The device allowed the detection of the SARS-CoV-2 antigen spike protein at concentrations as low as $1 \mathrm{fg} / \mathrm{mL}$ in phosphate buffer, a value much lower than that reported with ELISA and PCR methods [83]. The biosensor was tested in the universal transport medium (UTM), used for suspending the nasopharyngeal swabs for real clinical analysis. No reagent contained in UTM affected the measurements and the detection limit resulted to be $100 \mathrm{fg} / \mathrm{mL}$. In addition, the proposed COVID-19 sensor showed no significant response to MERS-CoV spike proteins, assuring high selectivity and specificity for the SARS-CoV-2 spike antigen protein. Finally, the performance of the sensor was tested in real clinical samples, collecting nasopharyngeal swab specimens of COVID-19 patients and of normal subjects. The COVID-19 FET-based nanobiosensor allowed to discriminate between patient and normal samples with detection limits lower than those reported with other current methods, without any sample preparation or preprocessing.

Noble metal nanostructures have been frequently used for virus detection systems to enhance functions such as specificity and sensitivity. A DNA-nanosensor for SARS-CoV-2 detection was recently proposed by Qiu and coworkers [76]. They realized a dual DNA-sensor consisting of a single chip, modified with a two-dimensional distribution of gold nanoislands (AuNIs). The chip integrates the plasmonic photothermal (PPT) effect and the localized surface plasmon resonance (LSPR) sensing transduction. The sensor chip was functionalized with complementary DNA (cDNA) receptors by forming $\mathrm{Au}-\mathrm{S}$ bonds between the AuNIs and the thiolic groups of cDNA. A schematic representation of the AuNIs PPT enhanced LSPR biosensor is shown in Fig. 5. The proper surface functionalization can suppress the non-specific binding events, thus increasing the sensitivity of the biosensor. The PPT heat, generated in situ on the same AuNI chip when illuminated at their plasmonic resonance frequency, was capable to significantly improve the kinetics and the specificity of the hybridization of SARS-CoV-2 nucleic acid sequences to their cDNA. A large number of false positive or false negative have been reported with current methods of COVID-19 detection. The PPT heating is capable of inhibiting the spurious binding of nonmatching sequences, thus avoiding an incorrect diagnosis. The dual-functional biosensor exhibited a linear range between $0.1 \mathrm{pM}$ and $1 \mathrm{mM}$ with a detection limit of $0.22 \mathrm{pM}$, which resulted low enough for direct analysis of SARS-CoV-2 sequences in respiratory real samples. Similar multiple nonspecific gene sequences from SARS-CoV and SARS-CoV-2 were tested and discriminated, attesting the high selectivity of the biosensor towards cross-reactive and interfering sequences. 
Fig. 3 Schematic representation of AuNPs immunosensor for MERS-CoV. List of abbreviations: Cys, cysteamine; Glu, glutaraldehyde; BSA, bovine serum albumin
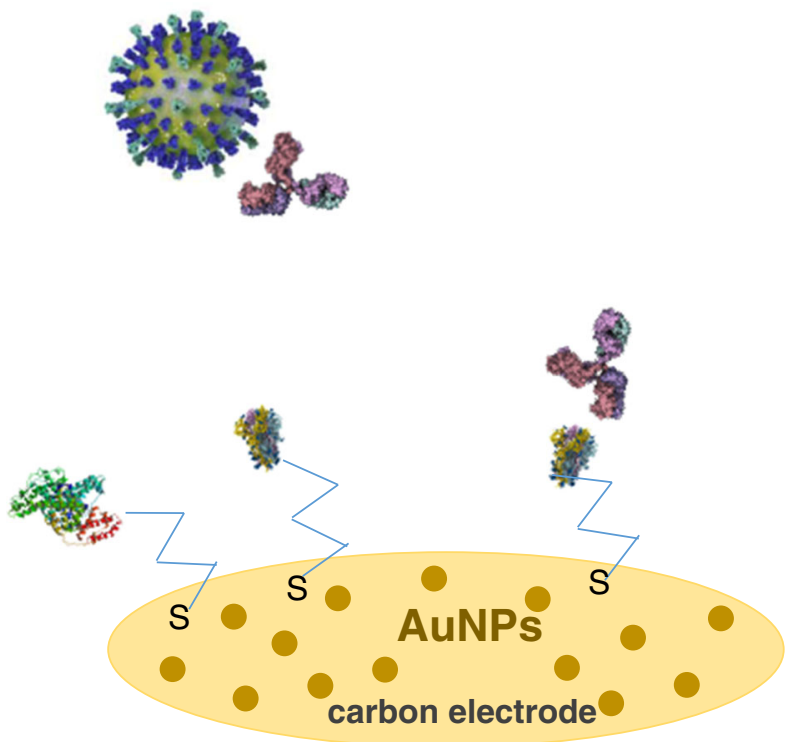

MERS-CoV

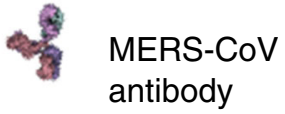

antibody

MERS-CoV spike protein S1

s Cys + Glu

BSA
The analytical performances of the reported COVID-19 nanobiosensors are summarized in Table 3.

Another promising tool for detection of SARS-CoV-2 viral genome is the Clustered Regularly Interspaced Short Palindromic Repeats (CRISP)-associated (Cas) enzymes technology, which allowed the detection of specific COVID19 gene sequences with detection limits between 10 and 100 copies per microliter in less than $1 \mathrm{~h}$ employing a target amplification (RPA) [84]. Recently, a research group has announced his interest to develop an amplification free electrochemical CRISP biosensor for on-site COVID-19 testing, with nanomodification of the electrode surface for signal enhancement [71].

Another recent study describes a portable graphene-based electrochemical biosensor for highly sensitive POC testing of Vibrio parahaemolyticus in seafood, which could be translated for COVID-19 detection. The detection was carried out using loop-mediated isothermal amplification (LAMP) and a graphene-based screen-printed electrode (SPE). The interaction between SPE and amplicons results in a shift in cathodic current, which stems from the intercalation of redox probe to double-stranded DNA. A portable mini potentiostat is used with SPE for on-site POC detection [85]. Most recently, PathSensors Inc. announced the development of a "Canary" fast biosensor for SARS-CoV-2 aerosol detection. The proposed platform utilizes a cell-based immunosensor that couples capture of the virus with signal amplification and provides a result in 3-5 min. PathSensors is based on a genetically engineered immune cell able to identify and bind to a specific target pathogen and then light up when the target pathogen is found. By measuring the light output from the cell, it is possible to know if the target pathogen is present in the sample. The initial application of the PathSensors device will be for testing of environmental swabs and air monitoring in sensitive spaces, such as hospitals, offices and food services. Validation data of the new biosensors will be available soon [72].
Fig. 4 Schematic representation of graphene FET-based immunosensor for SARS-CoV-2. List of abbreviations: S, source; $\mathrm{D}$, drain
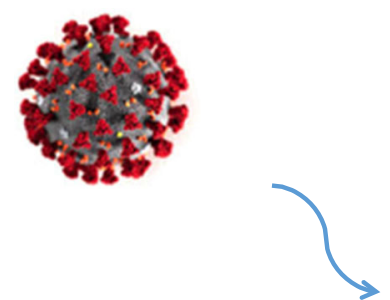

graphene

D

$\mathrm{Si} / \mathrm{SiO}_{2}$
SARS-CoV-2 spike protein

SARS-CoV-2 antibody 
Fig. 5 Schematic representation of AuNIs PPT-enhanced LSPR DNA-sensor. List of abbreviations: AuNIs, gold nanoislands; PPT, plasmonic photothermal; LSPR, localized surface plasmon resonance; cDNA, complementary DNA
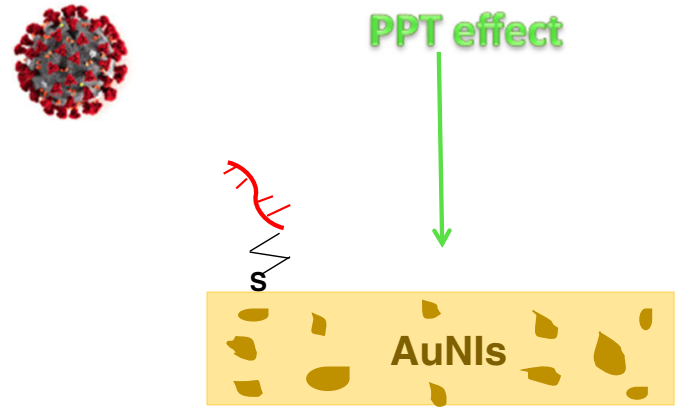

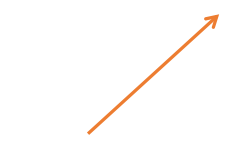

light source

detector

SARS-CoV-2 oligonucleotides

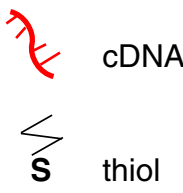

AuNI

\section{Antibody detection}

A different approach in the detection of COVID-19 infection is the development of nanobiosensors for anti-SARS-CoV-2 detection, after functionalization of the sensor's surface with the specific viral antigens.

Serologic assays for SARS-CoV-2 antibodies are now broadly available and play an important role in understanding the virus epidemiology in the general population and identifying groups at higher risk for infection. Unlike viral direct detection methods that can detect acutely infected persons, antibody tests are indirect tests that help determine whether the individual being tested was ever infected, even if that person never showed symptoms, by measuring the host humoral immune response to the virus. Therefore, serology antibody assays do not typically replace direct detection methods as the primary tool for diagnosing an active SARS-CoV-2 infection, but they do have several important applications in monitoring and responding to the COVID-19 pandemic. Demographic and geographic patterns of serologic antibody test results can help determine which communities may have experienced a higher infection rate and therefore may have higher rates of herd immunity. Moreover, serologic test results may assist with identifying persons potentially infected with SARS-CoV-2 and determining who may donate blood that can be used to manufacture convalescent plasma, as a possible treatment for COVID-19 disease.

Like infections with other pathogens, SARS-CoV-2 infection elicits the development of $\operatorname{IgM}$, IgA and IgG antibodies. IgA and IgM reach their peak during 4-25 days after illness onset, whereas IgG during 21-25 days, and therefore, they are used for diagnosis at early and late stages, respectively [73]. Anyhow, it remains uncertain how long the immunoglobulins remain detectable following infection and whether individuals with antibodies are protected against reinfection with SARS-CoV-2.
At present, no immuno- or DNA-sensors for the detection of immunoglubulins against SARS-CoV-2 are available on the market. Several nanobiosensors for the detection of specific antibodies against viral antigens or biomarkers have been extensively explored in literature, which could potentially being used for COVID-19 [86-88]. For example, a recent study has reported the development of a label-free electrochemical biosensor with an aptamer-functionalized black phosphorus (BP) nanostructured electrode [89]. The BP nanosheets are functionalized with anti-Ab-aptamers after coating with poly-L-lysine (PLL). BP-based biosensors show higher detection sensitivity and specificity compared to reduced graphene oxide biosensors, achieving detection limits down to pg level and ng level, respectively. A similar platform could be used also for highly sensitive detection of $\operatorname{IgG}$ or IgM against SARS-CoV-2 in patient blood samples.

\section{IoT biosensors}

An important issue regarding the use of nanobiosensors for early POC diagnosis and prevention of COVID-19 is their capability to upload collected data via a Bluetooth interface to an Android-based smartphone, which will successively transfer them to Health Centres Authorities to tackle the disease spread, as already realized by Zhou et al. [90], for an IoT (Internet of Things) real-time PCR system for dengue fever virus spread control.

The IoT applied to medicine, also called the Internet of Healthcare Things (IoHT), encompasses medical devices and software applications connected to the Internet, offering extensive healthcare services. The IoT has opened up a world of possibilities in the medical field: when connected to the Internet, ordinary medical devices can collect invaluable additional data, give extra insight into symptoms and trends, enable remote care, track medication orders and use wearable devices to transmit health information to the concerned health 
care professionals [91]. Future studies should further improve the function of smartphone and specific smartphone apps to enable on-site data analysis while allowing data storage to track patient health status.

By combining biosensors, artificial intelligence (AI), information technology and dynamic networking devices, IoT could provide long-distance communication between nanobiosensors, hospitals and patients, thus improving current medical conditions [92]. In Fig. 6 is a schematized IoT architecture of a next-generation nanobiosensor-based diagnostics system.

At present, an Internet of Things (IoT) based on a functional POC instrument for SARS-CoV-2 antibody detection is not available. The POC-measured biosensors data could be automatically uploaded via Bluetooth to the patient's smartphone or tablet and then sent through global communications to a central epidemiological data centre, for automated monitoring of the epidemiological situation. Obtained data could be made ready to fit into epidemiological models in order to forecast evolution of the epidemic outbreak. Suitable modelling algorithms have already been developed through AI to predict key parameters for clinical practitioner, polling IgG and IgM test results, to provide advanced diagnosis of individuals, to characterize the own progress of the illness and to help in categorizing patients, especially in the "transition zones", where the decision can be more doubtful (Fig. 6). Each validated diagnosis instance of a given patient, once anonymized, could be automatically transmitted to a central data station, for real-time advanced monitoring of the epidemics, epidemiological management, prevention of new virus outbreaks and evaluation of success of eventual vaccination. Recently, several contact tracing apps have been developed, thus offering technological solutions to the problem of controlling the spread of the virus and have worked reasonably well in countries such as Singapore, China, South Korea and other parts of Asia. However, in other countries, privacy concerns are limiting their introduction, which could limit efforts to control the pandemic [93].

\section{Conclusions and future perspectives}

The incorporation of nanomaterials into affinity-based biosensing applications has successfully demonstrated to allow fast, sensitive and reliable detection of SARS, MERS and

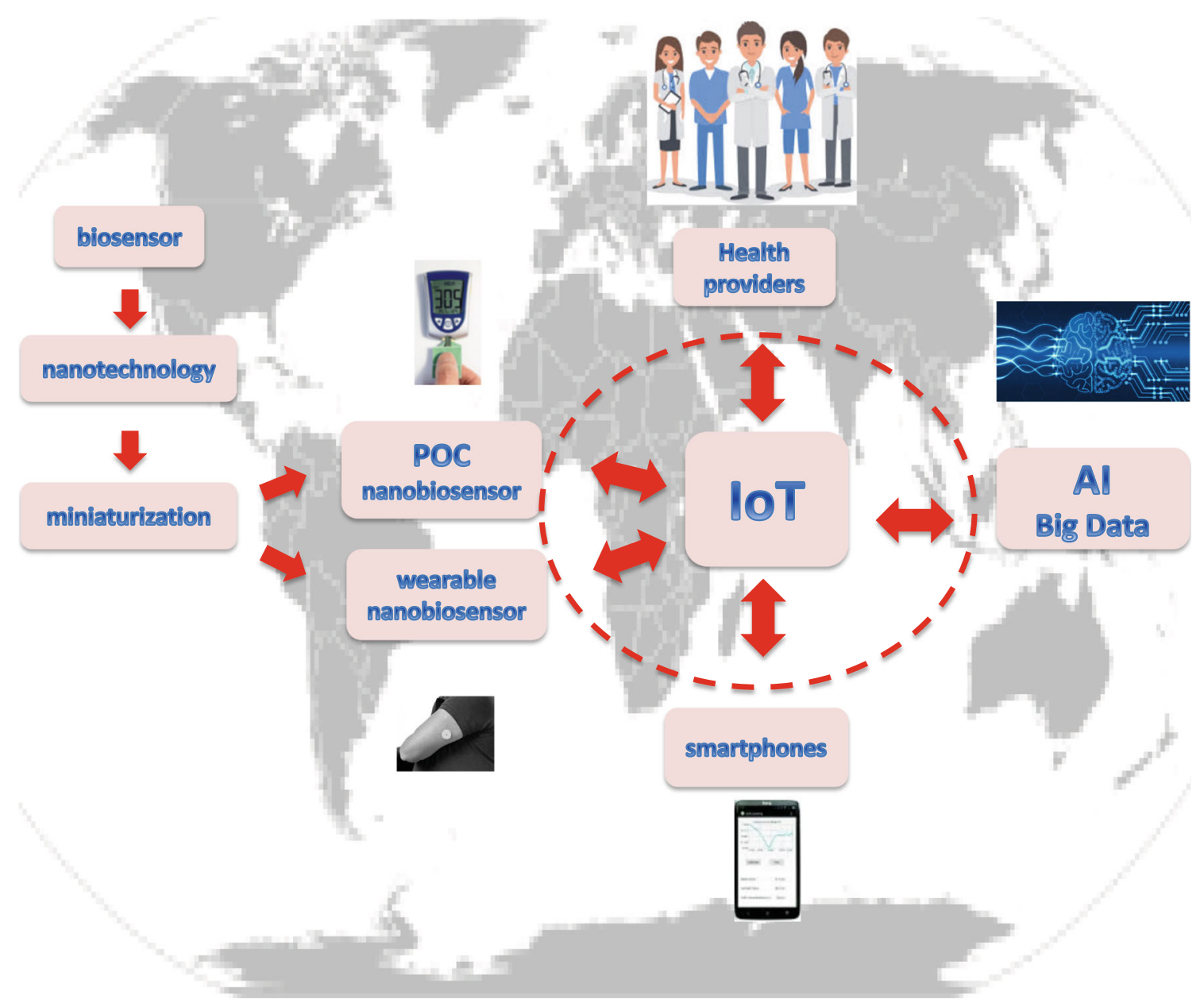

Fig. 6 Next-generation IoT nanobiosensor-based diagnostics system 
COVID-19. In particular, nanomaterial-based FET biosensors empowered the achievement of elevated biosensor performances, in terms of high sensitivity, selectivity and low detection limits. Graphene and $\operatorname{In}_{2} \mathrm{O}_{3}$ nanowires have been used for the exposed gated micro-region of the FET for very high electrochemical detection of SARS-CoV-2 and SARS-CoV, respectively. Other nanomaterials, such as gold nanoparticles and gold nanoislands, were successfully employed for the development of an immunosensor and a DNA-sensor for MERS and COVID-19, respectively, with detection limits in the fempto-pico molar range.

An important feature of the nanobiosensors with electrochemical and FET transduction is the possibility of their miniaturization into inexpensive and integrated platforms, similar in operation to handheld electrochemical readers, usable for POC diagnostics. Additionally, the affinity biosensors can be easily multiplexed, by incorporating multiple individually accessible electrodes on the same platform, thus allowing simultaneous detections. Of course, further efforts will be required before miniaturized POC multiplex testing practical applications are feasible.

Despite these encouraging properties, very few examples of nanobiosensors for SARS, MERS and COVID-19 detection have been developed and successfully applied in real clinical analysis so far. Of course, a lot of studies for COVID-19 are currently in progress. Point-of-care nanosensors for detection of SARS-CoV-2 antibodies are under development. At the moment, only lateral flow immunoassays produced by different companies are commercially available as single-use POC tests for anti-SARS-CoV-2 antibodies [94]. They are paper-like membrane strips coated with gold nanoparticle-antigen conjugates in the conjugation pad and antigens in the nitrocellulose membrane. A blood drop of the patient is deposited on the sample pad and is moved across the test by capillary action. Immobilized antibodies recognize and bind all human $\operatorname{IgG}$ and IgM. However, only human IgG-IgM/gold nanoparticle-antigen conjugates will produce a visible coloured line.

A next issue in nanobiosensing design should be the integration of the extraction system into the proposed biosensor to make it wearable and user-friendly. One possibility would be to translate the developed platforms into microneedles-based biosensors, which allow a continuous monitoring of SARS-CoV-2 antigens, antibodies or nucleic acid in the dermal interstitial fluid of both symptomatic and asymptomatic population. These wearable devices would provide important information on the following: (i) prevalence of infection in the community; (ii) development and decay of immunity in a population (the dynamics of "herd" immunity). As with individuals, the levels of antibody change over time (whether from natural infection or vaccination) and continuous measurements allow this to be tracked and potentially predict the likelihood of successive waves of the pandemic; (iii) post vaccination immune response, possibly indicating the need for a "booster" vaccination.
In summary, significant challenges are yet to be addressed and a lot of effort is worth to be invested in future studies for the development of IoT wearable nanobiosensors for COVID-19 detection, thanks to their great potential to perform rapid, accurate and in situ early diagnosis and, more importantly, to track the infectious diseases, thus preventing further pandemic outbreaks.

Acknowledgements The author thanks the Sapienza University of Rome for financial support.

Funding Open access funding provided by Università degli Studi di Roma La Sapienza within the CRUI-CARE Agreement.

\section{Compliance with ethical standards}

Conflict of interest The author declares that she has no competing interest.

Open Access This article is licensed under a Creative Commons Attribution 4.0 International License, which permits use, sharing, adaptation, distribution and reproduction in any medium or format, as long as you give appropriate credit to the original author(s) and the source, provide a link to the Creative Commons licence, and indicate if changes were made. The images or other third party material in this article are included in the article's Creative Commons licence, unless indicated otherwise in a credit line to the material. If material is not included in the article's Creative Commons licence and your intended use is not permitted by statutory regulation or exceeds the permitted use, you will need to obtain permission directly from the copyright holder. To view a copy of this licence, visit http://creativecommons.org/licenses/by/4.0/.

\section{References}

1. Peeri NC, Shrestha N, Rahman MS, Zaki R, Tan Z, Bibi S, Baghbanzadeh M, Aghamohammadi N, Zhang W, Haque U (2020) The SARS, MERS and novel coronavirus (COVID-19) epidemics, the newest and biggest global health threats: what lessons have we learned? Int J Epidemiol 1:1-10

2. Xie M, Chen Q (2020) Insight into 2019 novel coronavirus-an updated interim review and lessons from SARS-CoV and MERSCoV. Int J Infect Dis 94:119-124

3. WHO, World Health Organization (2020) Situation Report) Coronavirus disease 2019 (COVID-19). WHO, Geneva, p 40

4. Peiris JSM, Guan Y, Yuen KY (2004) Severe acute respiratory syndrome. Nat Med 10:S88-S97

5. Peiris JSM (2012) Coronaviruses, In: Medical microbiology (8th edition), Churchill Livingstone, pp. 587-593

6. McBride R, Fielding BC (2012) The role of severe acute respiratory syndrome (SARS)-coronavirus accessory proteins in virus pathogenesis. Viruses 4:2902-2923

7. Zhong N, Zheng B, Li Y, Poon L, Xie X, Chan K et al (2003) Epidemiology and cause of severe acute respiratory syndrome (SARS) in Guangdong, People's Republic of China, in February. Lancet 25:1353-1358

8. Assiri A, Al-Tawfiq JA, Al-Rabeeah AA, Al-Rabiah FA, Al-Hajjar S, Al-Barrak A, Flemban H, Al-Nassir WN, Balkhy HH, Al-Hakeem RF, Makhdoom HQ, Zumla AI, Memish ZA (2013) Epidemiological, demographic, and clinical characteristics of 47 cases of Middle East respiratory syndrome coronavirus disease from Saudi Arabia: a descriptive study. Lancet Infect Dis 13:752-761 
9. Lai CC, Shin TP, Ko WC, Tang HJ, Hsueh RP (2020) Severe acute respiratory syndrome coronavirus 2 (SARS-CoV-2) and corona virus disease-2019 (COVID-19): the epidemic and the challenges. Int J Antimicrob Agents 55:105924

10. Paudel S, Dangal G, Chalise A, Bhandari TR, Dangal OJ (2020) The coronavirus pandemic: what does the evidence show? Nepal Health Res Counc 18:1-9

11. Udugama B, Kadhiresan P, Kozlowski HN, H N, Malekjahani A, Osborne M, Li VYC, Chen H, Mubareka S, Gubbay JB, Chan WCW (2020) Diagnosing COVID-19: the disease and tools for detection. ACS Nano 14:3822-3835. https://doi.org/10.1021/acsnano.0c02624

12. Wang L, Wang Y, Ye D, Liu Q (2020) Review of the 2019 novel coronavirus (SARS-CoV-2) based on current evidence. Int $\mathrm{J}$ Antimicrob Agents 55:105948. https://doi.org/10.1016/j. ijantimicag.2020.105948

13. Wu Z, McGoogan JM (2020) Characteristics of and important lessons from the coronavirus disease 2019 (COVID-19) outbreak in China: summary of a report of 72314 cases from the Chinese Center for Disease Control and Prevention. JAMA 323:1239-1242

14. Wu A, Peng Y, Huang B, Ding X, Wang X, Niu P, Meng J, Zhu Z, Zhang Z, Wang J, Sheng J, Quan L, Xia Z, Tan W, Cheng G, Jiang T (2020) Genome composition and divergence of the novel coronavirus (2019-nCoV) originating in China. Cell Host Microbe 27:325-328

15. Chan JF, To KK, Tse H, Jin DJ, Yuen KY, Tse H, Jin DY, Yuen KY (2013) Interspecies transmission and emergence of novel viruses: lessons from bats and birds. Trends Microbiol 21:544-555

16. Hu B, Ge X, Wang LF, Shi Z (2015) Bat origin of human coronaviruses. Virol J 12:221-231

17. Zhang T, WuQ, ZhangZ(2020) Probable pangolin origin ofSARS-CoV2 associated with the COVID-19 outbreak. Curr Biol 30:1346-1351

18. Liu Y, Gayle AA, Wilder-Smith A, Rocklov J (2020) The reproductive number of COVID-19 is higher compared to SARS coronavirus. J Travel Med 27:1-4

19. Huang C, Wang Y, Li X, Ren L, Zhao J, Hu Y (2020) Clinical features of patients infected with 2019 novel coronavirus in Wuhan, China. Lancet 395:497-506

20. Petrosillo N, Viceconte G, Ergonul O, Ippolito G, Petersen E (2020) COVID-19, SARS and MERS: are they closely related? Clin Microbiol Infect 26:729-734. https://doi.org/10.1016/j.cmi.2020.03.026

21. Signorelli C, Scognamiglio T, Odone A (2020) COVID-19 in Italy: impact of containment measures and prevalence estimates of infection in the general population. Acta Biomed 91(91):3S

22. Shen M, Zhou Y, Abdu JY, Ye J, Al-Maskri AAA, Kang J, Zeng S, Cai S (2020) Recent advances and perspectives of nucleic acid detection for coronavirus. J Pharm Anal 10:97-101

23. Bastos ML, Tavaziva G, Abidi SK, Campbell JR, Haraoui L-P, Johnston JC, Lan Z, Law S, edt al. (2020) Diagnostic accuracy of serological tests for covid-19: systematic review and meta-analysis. BMJ 370:m2516

24. Kashir J, Yaqinuddin A (2020) Loop mediated isothermal amplification (LAMP) assays as a rapid diagnostic for COVID-19. Med Hypoteses 141:109786. https://doi.org/10.1016/j.mehy.2020.109786

25. Gubala V, Harris LF, Ricco AJ, Tan MX, Williams DE (2012) Point of care diagnostics: status and future. Anal Chem 84:487-515

26. Nayak S, Blumenfeld NR, Laksanasopin T, Sia SK (2017) Point-ofcare diagnostics: recent developments in a connected age. Anal Chem 89:102-123

27. Carter LJ, Garner VV, Smoot JW, Li Y, Zhou Q, Saveson CJ, Sasso JM, Gregg AC, Soares DJ, Beskud TR, Jervey SR, Liu C (2020) Assay techniques and test development for COVID-19 diagnosis. ACS Cent Sci 6:591-605
28. Zhu H, Fohlerova Z, Pekàrek Z, Basova E, Neuzil P (2020) Recent advances in lab-on-a-chip technologies for viral diagnosis. Biosens Bioelectron 153:112041

29. Lafleur JP, Jönsson A, Senkbeil S, Kutter JP (2016) Recent advances in lab-on-a-chip for biosensing applications. Biosens Bioelectron 76:213-233

30. Mollarasouli F, Kurbanoglu S, Ozkan SA (2019) The role of electrochemical immunosensors in clinical analysis. Biosensors 86:86. https://doi.org/10.3390/bios9030086

31. Antiochia R, Favero G, Conti ME, Mazzei F, Tortolini C (2015) Affinity-based biosensors for pathogenic bacteria detection. Int $\mathrm{J}$ Environ Technol Manag 18:85-206

32. Zhang X, Guo Q, Cui D (2009) Recent advances in nanotechnology applied to biosensors. Sensors 9:1033-1053

33. Mokhtarzadeh A, Eivazzadeh-Keihan R, Pashazadeh-Panahi M, Hejazi N, Gharaatifar M, Hasanzadeh B, Baradaran de la Guardia MP (2017) Nanomaterial-based biosensors for detection of pathogenic virus. Trends Anal Chem 97:445-457

34. Holzinger M, Le Goff A, Cosnier S (2014) Nanomaterials for biosensing applications: a review. Front Chem. https://doi.org/10. 3389/fchem.2014.00063

35. Sanvicens N, Pastells C, Pascual N, Marco MP (2009) Nanoparticle-based biosensors for detection of pathogenic bacteria. Trends Anal Chem 28:1243-1251

36. Ju H, Zhang X, Wang J (2011) Nanomaterials for immunosensors and immunoassays. In: NanoBiosensing, Biological and Medical Physics, Biomedical Engineering. Springer, New York, pp 425-452

37. Bishop KJM, Wilmer CE, Soh S, Grzybowski BA (2009) Nanoscale forces and their uses in self-assembly. Small 5:1600-1630

38. Lee SH, Sung JH, Park TH (2012) Nanomaterial-based biosensor as an emerging tool for medical applications. Ann Biomed Eng 40:1384-1397

39. Singh P, Pandey SK, Singh J, Srivastava S, Sachan S, Singh SK (2016) Biomedical perspective of electrochemical nanobiosensors. Nano-Micro Lett 8:193-203

40. Lei J, Ju H (2012) Signal amplification using functional nanomaterials for biosensing. Chem Soc Rev 41:2122-2134

41. Putzbach W, Ronkainen N (2013) Immobilization techniques in the fabrication of nanomaterial-based electrochemical biosensors: a review. Sensors 13:4811-4840

42. Battigelli A, Ménard-Moyon C, Da Ros T, Prato M, Bianco A (2013) Endowing carbon nanotubes with biological and biomedical properties by chemical modifications. Adv Drug Deliv Rev 65:1899-1920

43. Kuila T, Bose S, Khanra P, Mishra AK, Kim NH, Lee JH (2011) Recent advances in graphene-based biosensors. Biosens Bioelectron 26:4637-4648

44. Algar WR, Tavares AJ, Krull UJ (2010) Beyond labels: a review of the application of quantum dots as integrated components of assays, bioprobes, and biosensors utilizing optical transduction. Anal Chim Acta 673:1-25

45. Li Y, Schluesener H, Xu S (2010) Gold nanoparticle-based biosensors. Gold Bull 43:29-41

46. Bollella P, Schultz C, Favero G, Mazzei F, Ludwig R, Gorton L, Antiochia R (2017) Green synthesis and characterization of gold and silver nanoparticles and their application for development of a third generation lactose biosensor. Electroanal 29:77-86

47. Pingarrón JM, Yáñez-Sedeño P, González-Cortés A (2008) Gold nanoparticle-based electrochemical biosensors. Electrochim Acta 53:5848-5866

48. Antiochia R, Bollella P, Favero G, Mazzei F (2016) Nanotechnology-based surface plasmon resonance affinity biosensors for in vitro diagnostics. Int J Anal Chem:2981931

49. Chinh VD, Speranza G, Migliaresi C, Chuc NV, Tan VM, Phuong N-T (2019) Synthesis of gold nanoparticles decorated with 
multiwalled carbon nanotubes (Au-MWCNTs) via cysteaminium chloride functionalization. Sci Rep 9:5667. https://doi.org/10. 1038/s41598-019-42055-7

50. Ozhikandathil J, Badilescu S, Packirisamy M (2012) Gold nanoisland structures integrated in a lab-on-a-chip for plasmonic detection of bovine growth hormone. J Biomed Opt 17:077001

51. Subramoney S (2006) Carbon nanotubes, In Encyclopedia of materials: science and technology (2nd edition), pp. 1-8

52. Wang J (2005) Carbon-nanotube based electrochemical biosensors: a review. Electroanal 17:7-14

53. Le Goff A, Holzinger M, Cosnier S (2011) Enzymatic biosensors based on SWCNT-conducting polymer electrodes. Analyst 136:1279-1287

54. Balasubramanian K, Burghard M (2006) Biosensors based on carbon nanotubes. Anal Bioanal Chem 385:452-468

55. Gruner G (2006) Carbon nanotube transistors for biosensing applications. Anal Bioanal Chem 384:322-335

56. Besteman K, Lee JO, Wiertz FGM, Heering HA, Dekker C (2003) Enzyme-coated carbon nanotubes as single-molecule biosensors. Nano Lett 3:727-730

57. Valentini F, Carbone M, Palleschi G (2013) Carbon nanostructured materials for applications in nano-medicine, cultural heritage, and electrochemical biosensors. Anal Bioanal Chem 405:451-465

58. Vamvakaki V, Chaniotakis NA (2007) Carbon nanostructures as transducers in biosensors. Sensors Actuators B Chem 126:193-197

59. Liu S, Guo X (2012) Carbon nanomaterials field-effect-transistorbased biosensors. NPG Asia Mater 4:e23

60. Zhu Z (2017) An overview of carbon nanotubes and graphene for biosensing applications. Nano Lett 9:25. https://doi.org/10.1007/ s40820-017-0128-6

61. Yang W, Ratinac KR, Ringer SP, Thordarson P, Gooding JJ, Braet F (2010) Carbon nanomaterials in biosensors: should you use nanotubes or graphene? Angew Chem Int Ed 49:2114-2138

62. Ma H, Wu D, Cui Z, Li Y, Zhang Y, Du B et al (2012) Graphene-based optical and electrochemical biosensors: a review. Anal Lett 46:1-17

63. Ratinac KR, Yang W, Gooding JJ, Thordarson P, Braet F (2011) Graphene and related materials in electrochemical sensing. Electroanal 23:803-826

64. Contreras-Naranjo JE, Aguilar O (2019) Suppressing non specific binding of proteins onto electrode surfaces in the development of electrochemical immunosensors. Biosensors 9:15

65. Wu G, King M, Meyappan M, Lai KWC (2018) Simulation of graphene field-effect transistor biosensors for bacterial detection. Sensors 18:1715. https://doi.org/10.3390/s18061715

66. Ko H, Lee S, Koo K, Kim K, Cho D (2018) Nanowire-based biosensors: from growth to applications. Micromachines 9:679. https:// doi.org/10.3390/mi9120679

67. Patolsky F, Zheng G, Lieber CM (2006) Nanowire-based biosensors. Anal Chem 78:4260-4269

68. Kaushika A, Kumarb R, Hueyb E, Bhansalib S, Naira N, Nanira M (2014) Silica nanowires: growth, integration, and sensing applications. Mikrochim Acta 181:1759-1780

69. Arora P, Sindhu A, Dilbaghi N, Chaudhury A (2013) Engineered multifunctional nanowires as novel biosensing tools for highly sensitive detection. Appl Nanosci 3:363-372

70. Ishikawa F, Chang HK, Curreli M, Liao H, Olson AC, Chen PO-C, Zhang R, Roberts RW, Sun R, Cote RJ, Thompson ME, Zhou C (2009) Label-free, electrical detection of the SARS virus N-protein with nanowire biosensors utilizing antibody mimics as capture probes. ACS Nano 3:1219-1224

71. Cardean Transistors, (2020) Technology Networks, WWW Document. https://www.technologynetworks.com/tn/productnews/cardean-transistors-made-available-to-companies-andgovernment-agencies-willng-to-build-handled-332759
72. PathSensors, Inc. (2020) Announced the development of a SARSCoV2 biosensor. PathSensors Inc, PathSensors News and Press

73. Ma H, Zeng W, He H, Zhao D, Jiang D, Zhou P, Cheng L, Li Y, Ma $\mathrm{X}$, Jin $\mathrm{T}$ (2020) Serum IgA, IgA and IgMG responses in COVID19. Cell Molec Imm 17:773-775

74. Layqah L, Eissa S (2019) An electrochemical immunosensor for the corona virus associated with the Middle East respiratory syndrome using an array of gold nanoparticle-modified carbon nanotubes, Microchim. Acta 86:224. https://doi.org/10.1007/s00604-019-3345-5

75. Seo G, Lee G, Kim MJ, Baek S-H, Choi M, Ku KB, Lee C-S, Jun S, Park D, Kim HG, Kim S-J, Lee JO, Kim BT, Park EC, Kim SI (2020) Rapid detection of COVID-19 causative virus (SARS-CoV2 ) in human nasopharyngeal swab specimens using field-effect transistor-based biosensor. ACS Nano 4:5135-5142

76. Qiu G, Gai Z, Tao Y, Schmitt J, Kullak-Ublick GA, Wang J (2020) Dual-functional plasmonic photothermal biosensor for highly accurate severe acute respiratory syndrome coronavirus 2 detection. ACS Nano 14:5268-5277. https://doi.org/10.1021/acsnano.0c02439

77. Park TJ, Hyun MS, Lee HJ, Lee SY, Ko S (2009) A self-assembled fusion protein-based surface plasmon resonance biosensor for rapid diagnosis of severe acute respiratory syndrome. Talanta 79:295-301

78. Huang J, Chang C, Chen YF, Su L-C, Lee C-W, Chen C-C, Chen Y-MA, Chou C (2009) Detection of severe acute respiratory syndrome (SARS) coronavirus nucleocapsid protein in human serum using a localized surface plasmon coupled fluorescence fiber-optic biosensor. Biosens Bioelectron 25:320-325

79. Zuo B, Li S, Zhang J, Chen C (2004) Piezoelectric immunosensor for SARS-associated coronavirus in sputum. Anal Chem 76:3536-3540

80. Du L, Yang Y, Zhou Y, Lu L, Li F, Jiang S (2017) MERS-CoV spike protein: a key target for antivirals. Expert Opin Ther Targets 21:131-143

81. Wang N, Shi X, Jiang L, Zhang S, Wang D, Tong P et al (2003) Structure of MERS-CoV spike receptor-binding domain complexed with human receptor DPP4. Lancet 362:1353-1358

82. Chen Y, Kang Y, Chen H, Luk HK, Poon RW, Chan JF, Yuen KY, Xia N, Lau SK, Woo PC (2015) A sensitive and specific antigen detection assay for Middle East respiratory syndrome coronavirus. Emerg Microbes Infect 4:e26

83. Chu DKW, Pan Y, Cheng SMS, Hui KPY, Krishnan P, Liu Y, Ng DYM, Wan CKC, Yang P, Wang Q, Peiris M, Poon LLM (2020) Molecular diagnosis of a novel coronavirus (2019-nCoV) causing an outbreak of pneumonia. Clin Chem 66:549-555

84. Zhang, F., Abudayyehm Gootenberg, J.S. A protocol for detection of COVID-19 using CRISP diagnostics

85. Kampeera J, Pasakon P, Karuwan C, Arunrut N, Sappat A, Sirithammajak S, Dechokiattawan N, Sumranwanich T, Chaivisuthangkura P, Ounjai P, Chankhamhaengdecha S, Wisitsoraat A, Tuantranont A, Kiatpathomchai W (2019) Pointof-care rapid detection of Vibrio parahaemolyticus in seafood using loop-mediated isothermal amplification and graphene-based screen-printed electrochemical sensor. Biosens Bioelectron 132: 271-278. https://doi.org/10.1016/j.bios.2019.02.060

86. Jin S-A, Marinero EE, Stanciu LAS, Poudyal S, Kuhn RJ (2017) Impedimetric Zika and dengue biosensor based on functionalized graphene oxide wrapped silica particles. Bull Am Phys Soc 62: 116-430. https://doi.org/10.1016/j.electacta.2016.02.116

87. Liu M, Zhang Q, Brennan JD, Li Y (2018) Graphene-DNAzymebased fluorescent biosensor for Escherichia coli detection. MRS Commun 8:687-694. https://doi.org/10.1557/mrc.2018.97

88. Luo M, Fan T, Zhou Y, Zhang H, Mei L (2019) 2D black phosphorus-based biomedical applications. Adv Funct Mater 29: 1808306. https://doi.org/10.1002/adfm.201808306

89. Kumar V, Brent JR, Shorie M, Kaur H, Chadha G, Thomas AG et al (2016) Nanostructured aptamer-functionalized black phosphorus 
sensing platform for label-free detection of myoglobin, a cardiovascular disease biomarker. ACS Appl Mater Interfaces 8:22860 22868. https://doi.org/10.1021/acsami.6b06488

90. Zhu H, Podesva P, Liu X, Zhang H, Teply T, Xu Y, Chang H, Qian A, Lei Y, Li Y, Niculescu A, Iliescu C (2020) IoT PCR for pandemic disease detection and its spread monitoring. Sensors Actuators B 303:127098

91. Mujawar MA, Gohel H, Bhardwaj SK, Srinivasan S, Hickman N (2020) Nano-enabled biosensing systems for intelligent healthcare: towards COVID-19 management. Mat Today Chem 17:100306
92. Morales-Narvaez E, Diner C (2020) The impact of biosensing in a pandemic outbreak: COVID-19. Biosens Bioelectron 163:112274

93. Dans E (2020) We need to sort out the privacy issues with contact tracing apps if we are going to bring the pandemic under control, Forbes

94. Wu J-L, Tseng W-P, Lin C-H, Lee T-F. et al. (2020) Four point-ofcare lateral flow immunoassays for diagnosis of COVID-19 and for assessing dynamics of antibody responses to on page 13 .

Publisher's note Springer Nature remains neutral with regard to jurisdictional claims in published maps and institutional affiliations. 